\title{
Resistência de genótipos de milho para cultivo em várzeas subtropicais à lagarta-do- cartucho Spodoptera frugiperda
}

\author{
Resistance of corn genotypes for subtropical lowland cropping to fall armyworm \\ Spodoptera frugiperda
}

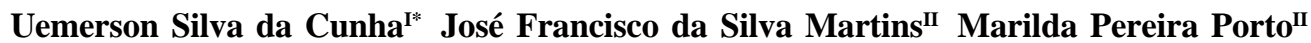 \\ Mauro Silveira Garcia ${ }^{\mathrm{I}}$ Oderlei Bernardi ${ }^{\mathrm{I}}$ Calisc de Oliveira Trecha ${ }^{\mathrm{I}}$ Daniel Bernardi \\ Edson de Oliveira Jardim ${ }^{\mathrm{I}}$ Evandro Carlos Uhlmann Back ${ }^{\mathrm{I}}$
} \begin{abstract}
elevado potencial para causar perdas econômicas na cultura do milho, no ecossistema de várzeas subtropicais. Com o objetivo de identificar fontes de resistência, foi avaliado, em laboratório, o desenvolvimento de S. frugiperda em nove genótipos de milho. Cem lagartas recém-eclodidas foram individualizadas em tubos de vidro, contendo porções de folhas de milho, mantidos à temperatura de $25 \pm 1^{\circ} \mathrm{C}$, umidade relativa de $70 \pm 10 \%$ e fotofase de $14 \mathrm{~h}$. Avaliaram-se a duração e a viabilidade das fases de larva e pupa, o peso de larvas e de pupas e a mortalidade larval. Dentre os genótipos avaliados, destacaram-se o BRS Missões-B e o BR 111 VI Sel. Dent C, os quais afetaram, respectivamente, o desenvolvimento da fase de larva e de pupa.
\end{abstract}

Palavras-chave: Zea mays, resistência de plantas, preferência.

\section{ABSTRACT}

Spodoptera frugiperda is the most important pest of the corn culture, causing high leaf losses. With intention to identify possible resistance sources, it was evaluated the effect of nine maize genotypes on the development of $\boldsymbol{S}$. frugiperda in laboratory. One hundred newly-hatched larvae were individualized in glass tubes containing leaves, maintained under temperature of $25 \pm 1^{\circ} \mathrm{C}$, relative humidity of $70 \pm 10 \%$ and photophase of $14 \mathrm{~h}$. Larval and pupal developmental time, survivorship and weight and larval mortality at 16 days were evaluated. It was observed that the genotypes BRS Missões- $B$ and BR 111 VI Sel. Dent $C$ affected the larval and pupal development, respectively.

Key words: Zea mays, host plant resistance, preference.
O cultivo de milho em várzeas subtropicais é uma das melhores alternativas para rotação de cultura com o arroz irrigado, principalmente para minimizar os níveis de infestação de plantas daninhas (GASTAL et al., 2004). Há evidências, porém, de que, devido às plantas de arroz e de milho serem hospedeiras para a lagarta-do-cartucho Spodoptera frugiperda (J.E. Smith, 1797) (Lepidoptera: Noctuidae) (PASHLEY et al., 1995; BUSATO et al., 2005), a proximidade de áreas com as duas culturas pode intensificar o ataque do inseto no milho.

A aplicação de inseticidas tem sido o principal método utilizado na tentativa de minimizar os prejuízos provocados pela lagarta-do-cartucho à cultura do milho (GASSEN, 1994). Muitas vezes, porém, além de tal prática não surtir o efeito esperado (GRÜTZMACHER et al., 2000), acarreta inconvenientes, como o aumento de riscos de contaminação ambiental e a elevação de custos devido a problemas com a tecnologia de aplicação, pela dificuldade de atingir as lagartas no interior do cartucho (GASSEN, 1994). Portanto, perante esta situação, aumenta a importância do uso de métodos alternativos para controle de $\boldsymbol{S}$. frugiperda em milho, como o uso de cultivares resistentes a insetos (VENDRAMIM \& FANCELLI, 1988) que possuam, além de características agronômicas desejáveis, resistência a esta praga

IDepartamento de Fitossanidade, Faculdade de Agronomia “Eliseu Maciel”, Universidade Federal de Pelotas (UFPel), CP 354, 96010-900, Pelotas, RS, Brasil. E-mail: uscunha@yahoo.com.br. *Autor para correspondência.

IIEmbrapa Clima Temperado, Pelotas, RS, Brasil. 
(CARBONARI et al., 1998). Neste contexto, a resistência de genótipos de milho à lagarta-do-cartucho foi avaliada em condições de laboratório.

Nove genótipos de milho para cultivo em várzeas subtropicais, obtidos na Estação Experimental de Terras Baixas da Embrapa Clima Temperado, foram avaliados, sendo identificados como BRS Missões-A, BRS Missões-B, BR 111 VI Sel. Dent C, GT-8C, GT-9C, ME 9002-24-9-3-1, M. Bonito-B E, PFMVS 95220 GeRS 320 B. Os genótipos foram cultivados em casa-devegetação, de acordo com as recomendações técnicas para a cultura do milho no Estado do Rio Grande do Sul (REUNIÃO, 2005). O genótipo ME 9002-24-9-3-1 foi utilizado como padrão suscetível por ter sido altamente infestado pelo inseto, conforme observado no campo.

Diariamente, as lagartas, oriundas de criação mantida em dieta artificial, foram alimentadas com folhas novas (estádios V8 a V10) dos genótipos de milho. Para cada genótipo, foram individualizadas 100 lagartas recém-eclodidas em tubos de vidro (2,5cm de diâmetro x 8,0cm de altura), contendo porções de folhas de milho de aproximadamente $12 \mathrm{~cm}^{2}$. Os tubos foram tamponados com algodão hidrófugo e mantidos em câmara climatizada à temperatura de $25 \pm 1^{\circ} \mathrm{C}$, umidade relativa de $70 \pm 10 \%$ e fotofase de $14 \mathrm{~h}$.

Os seguintes parâmetros biológicos foram avaliados: duração e viabilidade das fases de larva e pupa, peso de larvas e de pupas e mortalidade de larvas aos 16 dias após a infestação (DAI). O delineamento experimental foi inteiramente casualizado com 25 repetições, cada uma com quatro tubos (uma lagarta por tubo), totalizando os 100 indivíduos por tratamento. Comparações foram efetuadas pelo teste de Tukey em nível de 5\% de significância. Devido à constatação de homogeneidade de variâncias (Teste de Hartley), a transformação de dados não foi realizada.

Os genótipos diferenciaram-se significativamente quanto ao peso de lagartas de $\boldsymbol{S}$. frugiperda aos $16 \mathrm{DAI}$, destacando-se o genótipo BRS Missões-B como menos favorável ao inseto, comparativamente ao ME 9002-24-9-3-1 (padrão suscetível). Ademais, a fase larval foi mais prolongada no BRS Missões-B do que no ME 9002-24-9-3-1 (Tabela 1). As diferenças na duração da fase larval de $\boldsymbol{S}$. frugiperda podem ser atribuídas à qualidade de cada genótipo como alimento. De acordo com PARRA(1991), tanto a quantidade e a qualidade do alimento consumido por insetos, na fase larval, afetam, entre outros aspectos, a duração do ciclo biológico. Por outro lado, não foram detectadas diferenças significativas entre os genótipos BRS Missões-B e ME 9002-24-9-3-1 quanto à mortalidade e à viabilidade larval, nem mesmo entre os demais genótipos avaliados (Tabela 1).

Não foram detectadas diferenças significativas entre os genótipos quanto ao peso de pupas fêmeas. No entanto, pupas machos, derivadas de lagartas alimentadas no genótipo GT-9C, foram mais pesadas do que as pupas derivadas dos genótipos BRS Missões-A, RS 320 B e BR 111 Sel. Dent C, sem, no entanto, serem significativamente menos pesadas do que pupas machos oriundas de lagartas alimentadas no genótipo suscetível (Tabela 2). A alimentação de lagartas de $\boldsymbol{S}$. frugiperda no genótipo BR 111 VI Sel. Dent $C$, mesmo não tendo ocasionado efeitos deletérios significativos sobre a fase larval, reduziu a viabilidade de pupas (Tabela 2), diferindo dos genótipos BRS Missões-B, PFMVS 95220 G e RS 320 e padrão suscetível. Este resultado pode estar evidenciando

Tabela 1 - Duração, mortalidade, peso e viabilidade de larvas de Spodoptera frugiperda submetidas à alimentação em genótipos de milho, para cultivo em várzeas subtropicais.

\begin{tabular}{|c|c|c|c|c|}
\hline \multirow{2}{*}{ Genótipo } & \multirow[b]{2}{*}{ Duração (dias) } & \multirow[b]{2}{*}{ Mortalidade $^{1}(\%)$} & \multirow[b]{2}{*}{ Peso $^{1}$ (mg) } & \multirow[b]{2}{*}{ Viabilidade (\%) } \\
\hline & & & & \\
\hline BRS Missões-B & $23,3 \mathrm{a}$ & $34,0 \mathrm{a}$ & $169,8 \mathrm{~d}$ & $53,0 \mathrm{a}$ \\
\hline BRS Missões-A & $21,9 a b$ & $45,6 \mathrm{a}$ & $230,1 \mathrm{~cd}$ & $48,6 \mathrm{a}$ \\
\hline PFMVS $95220 \mathrm{G}$ & 21,3 bc & 33,6 a & $272,1 \mathrm{abc}$ & $47,3 \mathrm{a}$ \\
\hline ME 9002-24-9-3-1 (padrão) & 20,7 bcd & $45,4 \mathrm{a}$ & 293,2 abc & $60,3 \mathrm{a}$ \\
\hline GT-8 C & $19,8 \mathrm{~cd}$ & $50,6 \mathrm{a}$ & 279,4 abc & $47,0 \mathrm{a}$ \\
\hline GT-9 C & $19,4 \mathrm{~d}$ & $46,7 \mathrm{a}$ & 310,8 a & $47,7 \mathrm{a}$ \\
\hline RS 320 B & $19,4 \mathrm{~d}$ & 33,6 a & $296,3 \mathrm{ab}$ & 58,9 a \\
\hline BR 111 VI Sel. Dent C & $19,6 \mathrm{~d}$ & $43,6 \mathrm{a}$ & 246,7 bc & $53,0 \mathrm{a}$ \\
\hline M. Bonito-B E & $19,2 \mathrm{~d}$ & 36,6 a & $303,5 \mathrm{ab}$ & 56,9 a \\
\hline CV (\%) & 8,5 & 68,0 & 26,5 & 47,5 \\
\hline
\end{tabular}

${ }^{1}$ Mortalidade e peso de larvas registrados 16 dias após a infestação (16 DAI).

Médias não seguidas por mesma letra diferem entre si pelo teste de Tukey em nível de 5\% de significância. 
Tabela 2 - Peso (mg) das pupas de machos e fêmeas e viabilidade (\%) de pupas de Spodoptera frugiperda alimentadas com genótipos de milho.

\begin{tabular}{|c|c|c|c|}
\hline \multirow[t]{2}{*}{ Genótipo } & \multicolumn{2}{|c|}{---------------------Peso (mg)-------------------- } & \multirow{2}{*}{ Viabilidade (\%) } \\
\hline & Fêmea & Macho & \\
\hline BRS Missões-B & 159,6 a & $168,5 \mathrm{ab}$ & 87,5 a \\
\hline BRS Missões-A & 155,9 a & $153,9 \mathrm{~b}$ & $61,2 \mathrm{ab}$ \\
\hline PFMVS $95220 \mathrm{G}$ & 173,7 a & $166,0 \mathrm{ab}$ & 80,5 a \\
\hline ME 9002-24-9-3-1 (padrão) & 155,2 a & $165,3 \mathrm{ab}$ & 91,4 a \\
\hline GT-8 C & $153,2 \mathrm{a}$ & $163,4 \mathrm{ab}$ & $74,0 \mathrm{ab}$ \\
\hline GT-9 C & $171,1 \mathrm{a}$ & 178,7 a & $66,0 \mathrm{ab}$ \\
\hline RS 320 B & 152,2 a & 155,2 b & 82,3 a \\
\hline BR 111 VI Sel. Dent C & 145,8 a & $153,0 \mathrm{~b}$ & $49,9 \mathrm{~b}$ \\
\hline M. Bonito-B E & 150,6 a & $167,4 \mathrm{ab}$ & $74,4 \mathrm{ab}$ \\
\hline CV (\%) & 17,8 & 11,6 & 44,6 \\
\hline
\end{tabular}

Médias não seguidas por mesma letra diferem entre si pelo teste de Tukey em nível de 5\% de significância.

algum efeito cumulativo do alimento oferecido às lagartas de $\boldsymbol{S}$. frugiperda, conforme observado por VIANA \& POTENZA (2000). Estes autores, ao constatarem que genótipos mais atrativos e consumidos, em geral, foram menos adequados à biologia de $\boldsymbol{S}$. frugiperda, induziram que, primeiramente, há um efeito da planta hospedeira no comportamento do inseto que difere da adequação de cada genótipo ao balanço nutricional da lagarta.

A redução da viabilidade de pupas de $\boldsymbol{S}$. frugiperda, no genótipo BR 111 VI Sel. Dent C, pode estar associada a algum mecanismo de resistência que interferiu negativamente na nutrição das lagartas, pois, segundo SCRIBER \& SLANSKY JR. (1981), a fisiologia, o comportamento, a ecologia e a evolução de um inseto são afetados por fatores nutricionais. Neste sentido, segundo ORTEGA et al. (1980), é de fundamental importância elucidar os mecanismos de resistência envolvidos na relação planta/insetos, o que poderá facilitar a seleção de plantas com essa característica, aumentando a eficiência de programas de melhoramento genético. Considerando-se a atual situação de escassez de informações sobre resistência de milho, para cultivo em várzeas subtropicais, à $\boldsymbol{S}$. frugiperda, os resultados obtidos neste trabalho (Tabelas 1 e 2) constituem-se em subsídios importantes sobre o assunto. Destacam-se, principalmente, os genótipos BRS Missões-B e BR 111 VI Sel. Dent C, que poderão servir de base a futuros trabalhos sobre resistência de milho à lagarta-do-cartucho para cultivo em várzeas subtropicais.

\section{REFERÊNCIAS}

BUSATO et al. Biologia comparada de populações de Spodoptera frugiperda (J.E.Smith) (Lepidoptera: Noctuidae) em folhas de milho e arroz. Neotropical Entomology, v.34, n.5, p.743-750, 2005.

CARBONARI, J.J. et al. Reação de cultivares de milho ao ataque da lagarta-do-cartucho no agroecossistema de várzea. In: REUNIÃO TÉCNICA ANUAL DO MILHO, 43.; REUNIÃO TÉCNICA ANUAL DO SORGO, 26., 1998, Veranópolis, RS. Anais... Veranópolis: FEPAGRO, 1998. p.129-133.

GASTAL, M.F. da C. et al. Rotação e sucessão de culturas em áreas de várzea. In: GOMES, A. da S.; MAGALHÃES JR. A.M. (Eds.). Arroz irrigado no Sul do Brasil. Brasília: Embrapa Informação Tecnológica, 2004. Cap.23, p.799-830.

GASSEN, D.N. Pragas associadas à cultura do milho Passo Fundo: Aldeia Norte, 1994. 92p.

GRÜTZMACHER, A.D. et al. Insetos-pragas das culturas do milho e sorgo no agroecossistema de várzea. In: PARFITT, J.M.B. Produção de milho e sorgo em várzea. Pelotas: Embrapa Clima Temperado, 2000. p.87-102.

ORTEGA, A. et al. Breeding for insect resistance in maize. In MAXWELL, F.G.; JENNINGS, P.R. (Eds.). Breeding plants resistant to insects. New York: John Wiley \& Sons, 1980. p.371-420.

PARRA, J.R.P. Consumo e utilização de alimento por insetos. In: PANIZZI, A.R.; PARRA, J.R.P. (Ed.). Ecologia nutricional de insetos e suas implicações no manejo de pragas. São Paulo: Manole, 1991. p.9-65.

PASHLEY, D.P. et al. Host effects on developmental and reprodutive traits in fall armyworm strains (Lepidoptera: Noctuidae). Annals of the Entomology Society of América, v.88, n.6, p.748-755, 1995. 
REUNIÃO técnica anual de pesquisa de milho e sorgo do RS (50 e 33: 2005: Porto Alegre). Indicações técnicas para cultivo de milho e sorgo no Rio Grande do Sul 2005/2006. Porto Alegre: FEPAGRO/Emater-RS/ASCAR, 2005. 155p.

SCRIBER, J.M.; SLANSKY Jr., F. The nutritional ecology of immature insects. Annual Review of Entomology, v.26, p.183-211, 1981.
VENDRAMIM, J.D.; FANCELLI, M. Efeito de genótipos de milho na biologia de Spodoptera frugiperda (J.E. Smith, 1797) (Lepidoptera: Noctuidae). Anais da Sociedade Entomológica do Brasil, v.17, Supl, p.141-150, 1988.

VIANA, P.A.; POTENZA, M.R. Avaliação de antibiose e não-preferência em cultivares de milho selecionados com resistência à lagarta-do-cartucho. Bragantia, v.59, n.1, p.2733, 2000. 